Provided for non-commercial research and education use. Not for reproduction, distribution or commercial use.

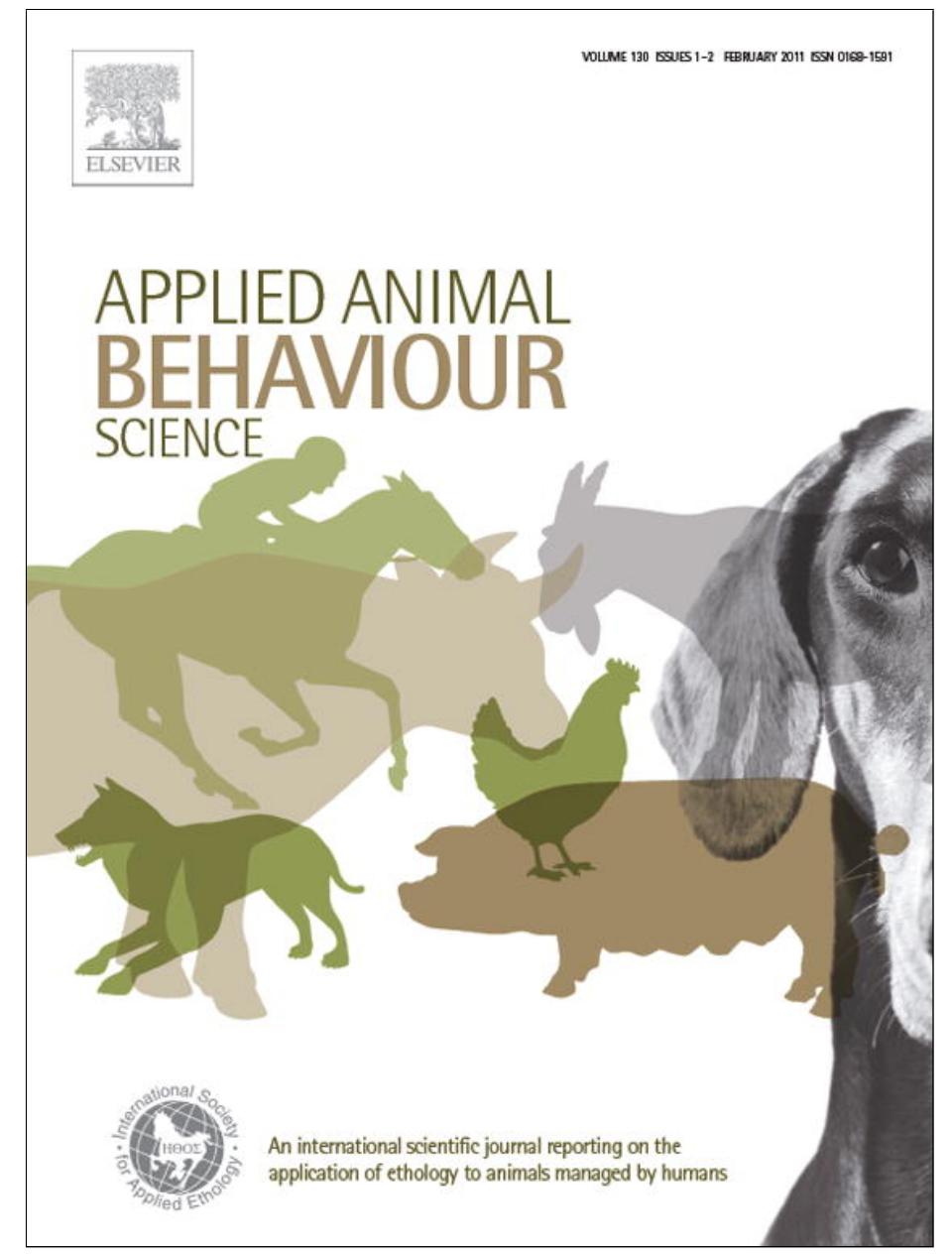

This article appeared in a journal published by Elsevier. The attached copy is furnished to the author for internal non-commercial research and education use, including for instruction at the authors institution and sharing with colleagues.

Other uses, including reproduction and distribution, or selling or licensing copies, or posting to personal, institutional or third party websites are prohibited.

In most cases authors are permitted to post their version of the article (e.g. in Word or Tex form) to their personal website or institutional repository. Authors requiring further information regarding Elsevier's archiving and manuscript policies are encouraged to visit:

http://www.elsevier.com/copyright 


\title{
Measuring motivation in a cichlid fish: An adaptation of the push-door paradigm
}

\author{
Leonor Galhardo ${ }^{\mathrm{a}, *}$, Olinda Almeida ${ }^{\mathrm{a}}$, Rui F. Oliveira ${ }^{\mathrm{a}, \mathrm{b}}$ \\ a Unidade de Investigação em Eco-Etologia, Instituto Superior de Psicologia Aplicada, Rua Jardim do Tabaco, 34, 1149-041 Lisboa, Portugal \\ b Champalimaud Neuroscience Programme, Instituto Gulbenkian de Ciência, Rua da Quinta Grande, 6, 2780-156 Oeiras, Portugal
}

\section{A R T I C L E I N F O}

\section{Article history:}

Accepted 13 December 2010

Available online 11 January 2011

\section{Keywords:}

Fish

Motivation

Push-door

Welfare

Tilapia

\begin{abstract}
A B S T R A C T
Recent behavioural, cognitive and neurophysiological studies strongly suggest that fish are capable of psychological experiences. Therefore, identifying needs from the animals' point of view is likely to be one of the best approaches to understand their welfare. Motivational tests, as a measure of what animals want, have been developed and refined for some decades. Despite numerous studies on fish motivational systems, none have attempted to quantify their motivation using this approach. Motivation studies often imply operant tasks for which various devices are used. The aim of this study was to adapt a push-door to quantify motivation in a cichlid fish, the Mozambique tilapia (Oreochromis mossambicus). Males of this species have strong snouts which they use for a number of activities and are thus suited to push. Twelve males of different social status were tested for three kinds of reinforcers: food, social partner and a control (additional space with substrate only). The animals were required to work the door (push/touch) at an ascending cost in order to have access to the resources. Measures of motivation included latency to open the door, work attention and maximum price paid. Latency to open the door increased with increasing cost for all resources, with the highest latency for the control reinforcer. Work attention was constant with increasing costs for social partners and food, and higher than the control. Work attention decreased for the control as cost increased. Maximum price paid was consistent with these results, being higher for social partners and food than for the control. The results of the three measures were consistent with each other and showed that the push-door can be used to measure motivation in this species. Further refinement of the present experimental set up will allow the use of this paradigm in the future, in order to improve knowledge on how this species values and ranks its needs.
\end{abstract}

(C) 2010 Elsevier B.V. All rights reserved.

\section{Introduction}

Fish welfare has been an area of growing interest and concern (Huntingford et al., 2006). Recent evidence on fish behavioural, neuroendocrine and cognitive processes suggests that they are capable of subjective feelings (reviewed in Galhardo and Oliveira, 2009). Since animal welfare

\footnotetext{
* Corresponding author. Tel.: +351 218811700; fax: +351 218860954 .

E-mail addresses: leonor_galhardo@ispa.pt, leonor.galhardo@mail.telepac.pt (L. Galhardo).
}

can be related to what animals feel (Dawkins, 1990), a knowledge of what they want has been regarded one of the most fruitful approaches to understand animals' perception of their own needs (Duncan, 2006; Kirkden and Pajor, 2006). This idea was recently extended to fish (Volpato et al., 2007). Based on analogical arguments, a clear link was established between an animals' needs, motivation and feelings: rewards they want to achieve (or avoid) may contribute to either lower suffering or increase pleasure (Dawkins, 1990; Widowski and Duncan, 2000; Kirkden and Pajor, 2006). Therefore, attempts to measure motivation are an indirect 
approach to understanding the subjective states of animals.

During the last three decades, methods from experimental psychology and the application of economic concepts have been used to measure motivation (Lea, 1978; Dawkins, 1983; Kirkden et al., 2003). Fish were never included in these studies, despite the fact that they also show motivational behaviour for certain resources. For example, goldfish exhibit trade-offs between the cost of a discrete shock and social contact (Dunlop et al., 2006) and feeding (Millsopp and Laming, 2008). Motivation tests are experimental procedures that aim to measure how much an animal is prepared to pay to have access to (or avoid) a given reinforcer (Kirkden and Pajor, 2006). Positive reinforcers used in these studies may be food, water, shelter, or many other species-specific enrichment items (Sherwin and Nicol, 1996; Mason et al., 2001; Hovland et al., 2006). Quantification of motivation requires the definition of an ascending reinforcement schedule per session, with costs being imposed on access to a given resource (e.g. Olsson and Keeling, 2002) or on its consumption (e.g. Dawkins, 1983). Costs may either involve price increases (e.g. weight of pushing doors, Petherick and Rutter, 1990) or income decreases (e.g. time available to spend with the resource, Dawkins, 1983). As costs increase, the animals' response may be measured as a function of work for access to the resource (e.g. Olsson et al., 2002; Hovland et al., 2006) or can be based upon consumption measures (e.g. Matthews and Ladewig, 1994; Hansen and Jensen, 2006). A quantifiable comparison among resources must involve an additional resource of known value (usually food) (Dawkins, 1983, 1990).

Motivation measurements are frequently based on a demand curve aiming to characterise consumption (demand or expenditure) as costs increase (Matthews and Ladewig, 1994). The elasticity of this consumption measures how much animals consider a resource a 'necessity' (inelastic demand) or a 'luxury' (elastic demand) (Dawkins, 1983). However, demand curves are not always considered the most valid measure of motivation. Economists, for example, do not use them to assess resource value, but rather the 'consumer surplus' (the area under the demand curve). Therefore, in animal motivation studies, the demand curves have been an inappropriately borrowed index from economists which require that animals have exclusive access to the resource under the test environment (closed economies), that the cost and resource-use vary proportionally and that the price per unit of resource is kept constant (Mason et al., 1998b; Kirkden and Pajor, 2006). Under different circumstances, instead of using elasticity of demand, it has been proposed to use the maximum price paid for a single access to the resource (Olsson and Keeling, 2002; Kirkden and Pajor, 2006).

Push-doors, as operant devices to study motivation, were first used in hens by Duncan and Kite (1987), where increased cost was imposed by adding weights to the door. This paradigm was further developed by Petherick and Rutter (1990) and used by a number of researchers (e.g. Olsson et al., 2002; Cooper and Appleby, 2003). Examples of operant studies in fish, although outside the context of motivational studies, include pendulum-pressing in rain- bow trout (Yue et al., 2008), switch-pulling in Nile tilapia (Endo et al., 2002) and rod-pushing in tench (Herrero et al., 2005) for food delivery. Earlier examples include operant tasks for access to food and mirrors for agonistic displays in Siamese fighting fish (Hogan et al., 1970).

The aim of the present study was to adapt the push-door paradigm to quantify motivation in the cichlid fish Mozambique tilapia. Males of this species have very strong snouts, which they use to build pits in the substrate and during mouth-to-mouth fights (Oliveira and Almada, 1998), and they easily learn conditioning paradigms (Antunes and Oliveira, 2009). We hypothesized that they could learn an operant task related to a push-door placed between them and a resource. Our primary aim was to test the usefulness of push-doors for studying motivation in this species, rather than to establish a comprehensive identification of resources' value.

\section{Materials and methods}

\subsection{Animals and housing}

This study involved the use of 12 males (weight $115 \pm 9.6 \mathrm{~g}$ ) of the species Oreochromis mossambicus. Their social status was identified prior to the experiment, six being territorial and six being non-territorial. Territorial males adopt a specific nuptial black coloration and a frequent territorial behaviour which includes nest building and its defence (Oliveira and Almada, 1996). They were part of a stock held at ISPA and were maintained in glass tanks $(120 \mathrm{~cm} \times 40 \mathrm{~cm} \times 50 \mathrm{~cm}, 240 \mathrm{l})$ with a fine gravel substrate, in stable social groups of 3-5 males and 5-6 females. The stock temperature was held at $26 \pm 2{ }^{\circ} \mathrm{C}$, with a $12 \mathrm{~L}: 12 \mathrm{D}$ photoperiod. Tanks were supplied with a double filtering system (sand and external biofilter, Eheim) and constant aeration. Water quality was analysed weekly for nitrites $(0.2-0.5 \mathrm{ppm})$, ammonia $(<0.5 \mathrm{ppm})$ (Pallintest kit $\left.{ }^{\circledR}\right)$ and $\mathrm{pH}$ (6.0-6.2). Fish were fed twice a day, $1-2 \%$ of their body weight with commercial cichlid floating and sinking sticks (ASTRA). The latter started to be given to the animals one month before the beginning of the experiment.

\subsection{Apparatus and push-door}

The experimental aquarium $(100 \mathrm{~cm} \times 40 \mathrm{~cm} \times 50 \mathrm{~cm}$, 200 1; Fig. 1a) was divided in two distinctive compartments: the start compartment ([SC], $40 \mathrm{~cm} \times 40 \mathrm{~cm} \times 50 \mathrm{~cm}$ ), and the resource compartment ([RC], $60 \mathrm{~cm} \times 40 \mathrm{~cm} \times 50 \mathrm{~cm}$ ). The SC was a fully barren compartment. The RC had a $5 \mathrm{~cm}$ layer of substrate (fine gravel) in area $\mathrm{RC}_{1}$ as a baseline environmental feature. Substrate was not placed in area $R_{0}$ to avoid interference with the door functioning. The female used for the social partner testing was placed in a smaller perforated aquarium $(12 \mathrm{~cm} \times 20 \mathrm{~cm} \times 50 \mathrm{~cm})$ in front of the door ( $20 \mathrm{~cm}$ of distance) (Fig. 1a). A fixed and opaque partition with a transparent swing-door $(17 \mathrm{~cm} \times 15.5 \mathrm{~cm})$ hinged in the centre separated the two compartments (Fig. 1b). The swing-door was made of Plexiglas and perforated to facilitate its movement. The fish were able to see the resources in the RC and to touch and push the access door for variable periods of time. Occasionally, some fish 


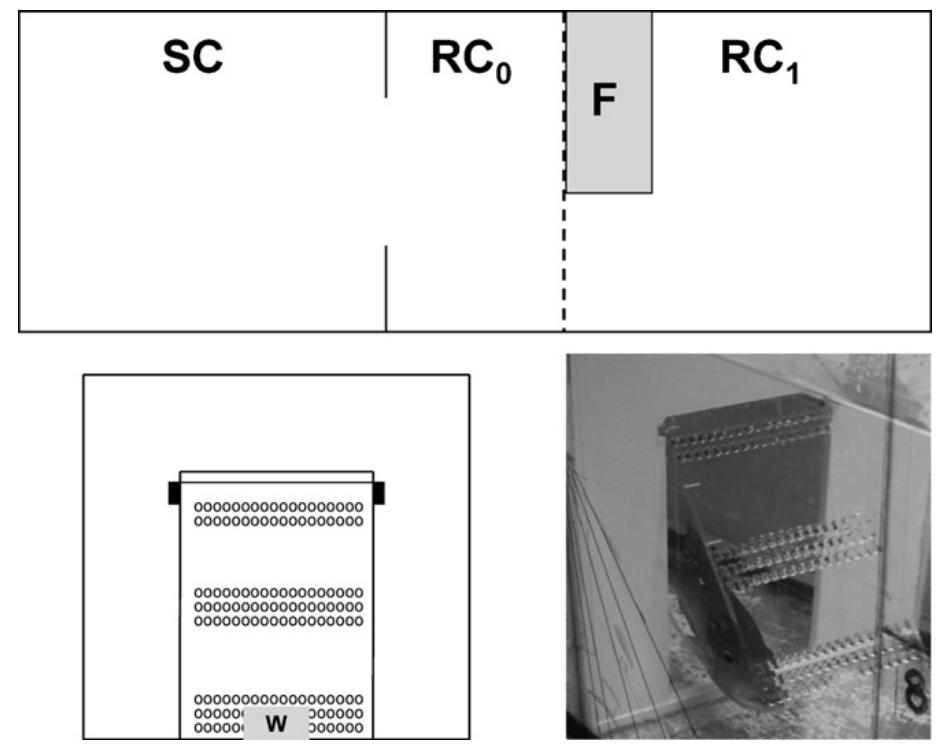

Fig. 1. (a) Schematic representation of the experimental set up (SC) start compartment $\left(\mathrm{RC}_{0}\right)$ resource compartment, area without sand ( $\left.\mathrm{C}_{1}\right)$ resource compartment, area with sand. F - Female aquarium used in social partner trials. (b) Schematic representation of the push-door. W - area where weights were attached to. (c) Photo of a Mozambique tilapia passing the push-door.

pushed the door wide enough to pass under it. For practical reasons, the door of this experimental design was operated manually (as in Olsson and Keeling, 2002; Hovland et al., 2006), by a transparent nylon string attached to the door's bottom, which could be pulled from outside the visual field of the animals. Therefore, when the animals reached a given number of touches or pushes (see below reinforcement schedule), the string would be pulled and the door opened. After the animal passed through, the door would remain opened until the end of the trial. In order to increase the opening cost, different plumb weights were attached (adapted from Duncan and Kite, 1987) to the external and bottom area of the door (Fig. 1c). The sides and back of each aquarium were covered with opaque material to reduce external stimuli. Aeration was removed during the tests, and reintroduced in between tests and overnight.

\subsection{Experimental procedure}

\subsubsection{Reinforcers}

This experiment involved three kinds of reinforcers: food, social partner (female) and additional space/substrate (as a control). Food and social partner were presented in a counter-balanced order. The control was tested after the presentation of these two reinforcers. Due to laboratory restrictions, only five males (two dominants, three subordinates) were tested for the control.

The food reward was kept constant throughout the study and consisted of three pellets of commercial food for cichlids per trial (two trials per day, which completed the individual daily portion). Two different kinds of pellets were used to amplify the food attractiveness: one out of the group of three pellets - divided in two halves immediately sank and remained in front of the door (visual stimulus), while the other two remained floating. As the latter were the pellets usually given to the fish, their use aimed to ensure the olfactory and rewarding properties of more familiar food. The pellets were introduced to the set up after the animals' acclimation. Fish were induced to anticipate this event by exposure to the sight of a yellow container previously associated with food. Pellets were placed in the RC immediately after this exposure. A previous study has shown that Mozambique tilapia is able to anticipate the delivery of food when it is predictably signed (Galhardo et al., submitted). In fact, a visual cue $5 \mathrm{~min}$ before food delivery resulted in increased activity towards the front of the aquarium, interspersed with frequent surfacing in the area where food was usually placed. Despite the fact that the individual daily amount of food was delivered during the trials, the animals were considered to be tested in an open economy, i.e. with access to the reward outside the experimental set up because they could still eat food particles in the home tanks.

The social partner was also tested in an open economy as test males were in full contact with their social group until arrival to the set up (where they spent only $30 \mathrm{~min}$ in isolation prior to trials). After having passed the door for access to the social partner, fish were allowed to remain in the RC for 30 min with free access to the SC (away from social partner). This time spent with the reward (reward size) was a long enough period for animals not to devalue their partner due to artificial interruptions of social bouts (Mason et al., 1998a). The third reinforcer (additional space/substrate) was intended as a control. Although it is known that males value substrate (Galhardo et al., 2008, 2009), here the RC with substrate was simply used as a baseline comparison to the remaining resources.

\subsubsection{Reinforcement schedule}

Animals expressed their interest in the RC by frequency of touching and/or strength of pushes at the door. Touching the door was defined as the physical contact established between the tip of the fish's snout and the door surface, without moving the door. Pushing the door was essentially a similar behaviour (probably belonging to the same continuum) but was performed with variable strengths that 
Table 1

(a) Reinforcement schedules for touches (frequency) and pushes (door's weight). (b) Number $v s$. amplitude of pushes required to open the door at any cost.

\begin{tabular}{llc}
\hline (a) & & \\
\hline Price & Touches (No.) & Door weight (g) \\
\hline Level 1 & 20 & 0 \\
Level 2 & 40 & 25 \\
Level 3 & 50 & 50 \\
Level 4 & 60 & 100 \\
Level 5 & 70 & 200 \\
& & \\
(b) & & \\
\hline Pushes to open the door & Amplitude & Number \\
\hline & $<15^{\circ}$ & 12 \\
& $\geq 15^{\circ}<30^{\circ}$ & 3 \\
& $\geq 30^{\circ}<45^{\circ}$ & 2 \\
& $\geq 45^{\circ}$ & \\
& &
\end{tabular}

varied how far the door opened. Occasionally, a fish pushed so strongly that it was able to open and pass through the door without external intervention. When this was not the case the door was operated manually in accordance with the reinforcement schedule described below. Touch and push were two spontaneous behaviours which we decided to incorporate as measures of fish's work in order to keep the behavioural response to the task as natural and spontaneous as possible, thus avoiding complex training protocols which could interfere with motivation (Würbel, 2009).

We imposed a fixed ratio (fixed number of responses required to obtain one reinforcer) which consisted of five work levels (from easiest, level 1 to most difficult, level 5) of an ascending cost to have access to the test resource, as described in Table 1. The costs imposed during the course of this experiment are related to the two exhibited behaviours - touches and pushes - and were defined as described in Table 1a. Therefore, ascending costs included an increased frequency of touches required for the door to be opened (from 20 touches at the easiest level to 70 touches at the most difficult level) or increased weights attached to the door (from $0 \mathrm{~g}$ at the easiest level to $200 \mathrm{~g}$ which was double the fish's body weight at the most difficult level). As described in Table $1 \mathrm{~b}$, the animals were required to perform a given number of pushes depending on their amplitudes. Range of difficulty, whether in relation to the frequency of touches or weights attached to the door and number of pushes, were decided during a preliminary pilot study.

The animals had a total of $15 \mathrm{~min}$ to complete each trial, i.e. to have access to the $\mathrm{RC}$, but their responses (touches/pushes) were only considered if performed within five consecutive minutes. This fixed amount of time was chosen on the basis of a previous pilot study where animals tended to respond in behavioural bouts of approximately $5 \mathrm{~min}$. After that they seemed to lose attention in relation to the operant task, swimming around and performing behaviours such as surfacing, nipping or dragging at the bottom (moving with inferior jaw in permanent touch with the bottom). To avoid overestimating the meaning of touches at the push-door, we discounted from the total amount of touches at the door all those also performed in other surfaces (e.g. walls). Expressed in an equation:

$T_{D}=T_{\mathrm{TD}}-T_{S}$,

in which $T_{D}$ - number of touches at the door; $T_{\mathrm{TD}}$ - total number of touches at the door; $T_{S}$ - number of touches in other surfaces.

In summary, for each imposed cost, the weighted door would be opened as soon as the established number of touches or 'number $v s$. amplitude' pushes was reached.

\subsection{Training and trials}

As mentioned, the spontaneous behavioural response to the door was touching and pushing, occasionally causing it to open. Thus, training simply aimed to reinforce both behaviours in such a way that levels of their performance could become a measure of fish attention and effort (work) at the door and inherently a measure of the motivation to access the resource on the other side. Training was done through a combination of operant conditioning and shaping techniques (Prescott and Buchanan-Smith, 2003). The reinforcer was food. An initial training period lasted for approximately five days (approx. $1 \mathrm{~h}$ per day). This period aimed to acclimatise animals to the set up and door (wide and semi-opened). At this stage, fish hesitantly approached the semi-opened door, took some time to touch it and eventually passed through the lateral opening. The training method at this point was shaped according to each individual with the objective of leading all the animals to contact and pass the door. After this time, during which they generally became less fearful, six training sessions (in three days) were undertaken immediately prior to the trials. In the first training session the door was semi-opened and all animals passed to the RC without difficulties. The following training sessions always started with the door closed, with no weights attached (the same as the first cost level). During the three next training sessions, different animals still passed the door at different number of touches or pushes. If they would not touch or push the door an acceptable number of times, it was a semi-opened in order to avoid frustration and subsequent disconnection from the task (Olsson et al., 2002). However, animals that did not work enough to open the door within $15 \mathrm{~min}$ from the start of the 4th training session were removed from the study. We made the decision at sessions 5-6 that animals were already trained, on the basis of a consistent decrease in latency to open the door in relation to the first sessions. On the basis of these criteria four animals were removed from the experiment because they showed inconsistent behaviour and signs of stress. Stressed behaviour was characterised by excessive inactivity, stressed body colour pattern (dark stripes) and elevated ventilatory rate.

Trials started the day immediately after training. Each male was individually transferred from its home tank directly to the experimental set up just before the trial started for the food trials, and $30 \mathrm{~min}$ before for the social partner and control trials. This short period of isolation in the set up was intended to create a change of context, improving attractiveness for social partners, without 
generating stress (see Section 4 for further details). The acclimation period for the food trials never exceeded $3 \mathrm{~min}$, which was time enough for the animal to recover from being moved (as indicated by reduced ventilatory rate, nonstressed colour pattern and swimming around). After the acclimation period, the food was placed in the RC, or an opaque partition (placed next to the door) was lifted to establish visual contact with the female in the RC. Costs increased progressively from the minimum (level 1) to the maximum work level (level 5) in five consecutive trials as described above. Animals were tested twice a day for food, following the usual feeding routine, and once a day for social partner and control/substrate. The experiment was run in two separate blocks of five and seven males (all individually tested) with a balanced presentation of the reinforcers (the second group was not tested for the control). After the fish achieved the established cost to open the door and pass to the resource compartment, the door remained open for free access to both compartments. After each trial, the fish was removed from the set up and returned to its home tank. The animal was always captured in the SC, to where it swam freely or was gently conducted, in order to avoid the establishment of an aversive relationship with the RC.

\subsection{Behavioural sampling}

The behavioural sampling was focal and continuous (Martin and Bateson, 2007). The methodology followed to count and register the number of pushes and touches involved two people with different roles throughout the study. One was exclusively devoted to the task of counting number of touches/pushes, observing remaining behaviour and opening the door at the required moments. The other person was exclusively registering the behaviour described by the first observer.

\subsubsection{Motivation measures}

The use of consumption measures to infer motivation (and hence demand curves) was not considered valid in this study for three reasons: it was an open economy; there was a prolonged access to the social partner (and control) without associated costs; and non-substitute resources were being compared. Measures of motivation were therefore related to resource access and included latency to door opening, work attention and maximum price paid. Latency to door opening was the period of time between the trial start and the door opening and reflected the work performed in a given amount of time: the faster the work performed, the lower the latency. Work attention (\%) was calculated as the time spent working at the door (period of time between the first touch/push and the door's opening, discounting the periods not investing at the door) divided by the latency to door's opening. Expressed in an equation:

$\mathrm{WA}=T_{D} / L \times 100 \%$

in which WA - work attention; $T_{D}$ - time spent working at the door; $L$ - latency to door's opening. It expresses the proportion of time spent working at the door before it opened. This measure intended to evaluate the degree of attention paid to the door/RC, regardless of the behavioural strategy adopted to open it (touches, pushes or both). Maximum price paid is the maximum cost at which the door was opened.

\subsubsection{Behaviour in relation to reinforcers}

Despite the fact that no measures of consumption were used to assess motivation, an exploratory analysis of behaviour in relation to reinforcers was undertaken. After the animals have passed the door, the latency to start eating was measured. In relation to the social partner reinforcer, 'time close to female' (within $2 \mathrm{~cm}$ from the female's tank), 'time interacting with female' (mutual touching of the female's tank walls) and 'time interacting with substrate' (nipping and pit digging) were the sampled behaviours. The amount of time spent in the three different areas of the experimental set up was recorded for both social partner and control. These observations were undertaken during the first $10 \mathrm{~min}$ (out of $30 \mathrm{~min}$ ) in contact with the reinforcer.

\subsection{Experimental design and data analysis}

In summary, the experimental design involved 12 animals divided in two groups of 5 and 7 males. All animals were subjected to two reinforcement treatments (food and social partner) in a counter-balanced way. The control reinforcement was only tested in the group of 5 males, after the testing of the two other reinforcers. Treatments were undertaken in different consecutive days, with weights increasing as trials progressed for each reinforcer. Therefore, this design involved two repeated measures which are the reinforcers and the door's weight (cost).

Latency and work attention were both analysed using a repeated measures analysis of variance (two repeated factors: reinforcers [2, if social partner $v s$. food] and cost [1-5]). In the ANOVA model 10 dependent variables were then compared $(2 \times 5)$. Differences between trials involving the social partner and food reinforcers were analysed with all 12 animals. The categorical factor 'social status' (territorial/non-territorial) was added to this ANOVA model in order to assess its possible influence in the variation. Planned comparisons of least squares means were carried out when appropriate. All the analyses involving a third reinforcer (control) included only five animals (the first block of tested animals). When there were significant differences in the variances between conditions (Levene's Test), data were normalised using the transformations proposed by Zar (1984), namely log transformation for latencies (seconds) and arcsin transformation for work attention (percentages). Differences in the maximum price paid between social partner and food reinforcer were analysed using the Wilcoxon Matched Pairs Test. Differences in maximum price paid among the three reinforcers (including control) were analysed with a Friedman ANOVA $(n=5)$. All analyses after access to the RC also involved repeated measures ANOVAs (repeated factor: cost [levels 1-5]; categorical predictor: social status or area). A value of $P<0.05$ denoted significance in all statistical tests. All analysis was performed using the statistical package (StatSoft Inc., USA, 1984-2008). 


\subsection{Ethical note}

The experiments described were conducted in accordance to national legal standards on protection of animals used for experimental purposes and are part of a project approved by the national authorities (Ref. 30489, 29/11/2007).

\section{Results}

\subsection{Behavioural strategy for reinforcers access}

In general, there was a mixed exhibition of pushes and touches, but each animal tended to increase the performance of one of them and in this way reached the amount of work pre-established to open the door. When the reinforcer was food, $51 \%$ of the animals which passed the door opened it through pushes, and $49 \%$ through touches. For the social partner, touches prevailed with $81 \%$ of the animals, against $18 \%$ which opened the door by pushes. The control resource was never accessed through pushes since $100 \%$ of the animals which accessed the resource compartment did it by reaching the scheduled number of touches. Occasionally, some animals achieved the cost imposed simultaneously through the required number of touches and pushes.

\subsection{Measures of motivation}

Latency to open the door increased with cost for social partner and food (repeated measures ANOVA, $F_{(4,44)}=67.6$, $P<0.001$; Fig. 2a), with no differences between these reinforcers (repeated measures ANOVA, $F_{(1,11)}=1.8$, NS; Fig. 2a). The latency to access the control reinforcer (additional space/substrate) also increased with cost (repeated measures ANOVA, $F_{(4,16)}=19.8, P<0.001$; Fig. $2 \mathrm{a}$ ) and it was the resource to which the access latency was the highest (repeated measures ANOVA, Reinforcer $(n=5)$ : $F_{(2,16)}=36.1, P<0.001$; Planned comp. of squared means: food $v s$. control $F=43.8, P=0.003$; social partner $v s$. control $F=73.8, P<0.001$; Fig. 2a). There was no overall significant influence of social status in the latency to get access to a social partner or to food (repeated measures ANOVA, $F_{(4,40)}=0.3$, NS; Fig. 2 b). However, at the highest prices (levels 4 and 5 ) the latency of non-territorial males increased significantly in contrast with the behaviour of territorial males (planned comparisons of LS means, $P<0.001$; Fig. 2b).

Work attention (percentage of time spent working at the door in relation to the latency to the door's opening) to access a social partner or food was similar at any cost (average value of $77.5 \%$ for food and $78.2 \%$ for social partner; repeated measures ANOVA, Reinforcer $\times$ Price: $F_{(4,44)}=1.8$, NS; Fig. 3a). Work attention to get access to the control reinforcer (additional space/substrate) was lower than for the social partner and food (average value of $51.9 \%$; repeated measures ANOVA $(n=5): F_{(2,8)}=8.4, P=0.01$, Fig. 3a) and tended to decrease with cost (from $66.5 \%$ at FR1 to 32.4 at FR\%; repeated measures ANOVA $(n=5): F_{(4,16)}=1.6, P=0.08$, Fig. 3a). Social status tended to influence work attention to access a social partner or food (repeated measures ANOVA, $F_{(4,40)}=2.5, P=0.06$; Fig. 3b). Similarly to latency,
Table 2

Maximum price paid (average \pm standard error) by territorial and nonterritorial males for food $(n=12)$, social partner $(n=12)$ and $\operatorname{control}(n=5)$ as expressed by the weight of the door.

\begin{tabular}{llll}
\hline & Food & Social partner & Control \\
\hline Territorial $^{*}$ & $100 \pm 37 \mathrm{~g}$ & $170 \pm 29 \mathrm{~g}$ & $25 \pm 25 \mathrm{~g}$ \\
Non-territorial $^{* *}$ & $121 \pm 38 \mathrm{~g}$ & $100 \pm 32 \mathrm{~g}$ & $50 \pm 25 \mathrm{~g}$ \\
\hline
\end{tabular}

${ }^{*} N=6$ except for control $(N=2)$.

** $N=6$ except for control $(N=3)$

this is particularly clear at high access prices (in this case, level 4), where the work attention of non-territorial males decreased significantly in contrast with the behaviour of territorial males (planned comparisons of LS means, $P<0.05$, Fig. 3b).

There was no difference between maximum price paid to have access to a social partner or food (Wilcoxon matched pairs test, $Z=0.6$, NS [ $n=12$ ]; Table 2 ). Differences between territorial and non-territorial males were not statistically significant. Maximum price paid for access to the control reinforcer (additional space/substrate) is lower than for access to a social partner/food (Friedman ANOVA, $\chi^{2}[n=5 ; \mathrm{df}=2]=7.7, P<0.05$; Table 2).

\subsection{Behaviour with reinforcers}

All animals ate the food immediately after entering the $\mathrm{RC}$. There was no difference in latency to start eating the food with different access costs or between social status (repeated measures ANOVA: $F_{(4,40)}=0.8$, NS). All animals ate the available pellets without interruptions.

Area use after access to the reinforcer was compared for the social partner and the control (additional space/substrate). In both cases there were clear differences in area use, as time spent in the area with substrate $\left(R C_{1}\right)$ was higher than in the area without substrate $\left(R C_{0}\right)$, with or without females (repeated measures ANOVA, social partner $[n=12]: F_{(2,33)}=213.3, P<0.001$; substrate $[n=5]$ : $F_{(2,12)}=70.6, P<0.001$, Fig. 4). When the social partner reinforcer was tested, males hardly visited the start compartment (SC), while when there was no social partner (control reinforcer) there was no pattern in $\mathrm{SC}$ and $\mathrm{RC}_{0}$ area use.

After having passed the door, time spent close to the female was the same regardless of the cost paid and social status (average of time spent close to the female along the five different costs: $32.8 \pm 2.9 \%$; repeated measures ANOVA: $F_{(4,40)}=1.2$, NS). However, territorial males spent more time interacting with the female than nonterritorial males (average of time interacting with the female along the five different costs: territorial, $29.0 \pm 4.2 \%$; non-territorial, $22.9 \pm 3.4 \%$; repeated measures ANOVA: $\left.F_{(1,10)}=6.8, P=0.03\right)$. There was no difference in male 'time interacting with substrate' along the different access costs (repeated measures ANOVA: $F_{(4,40)}=0.55$, NS) and between social status when the reinforcer was the social partner (repeated measures ANOVA: $F_{(1,10)}=1.71$, NS) and when it was only substrate/control (repeated measures ANOVA: $F_{(4,12)}=0.62$, NS). 

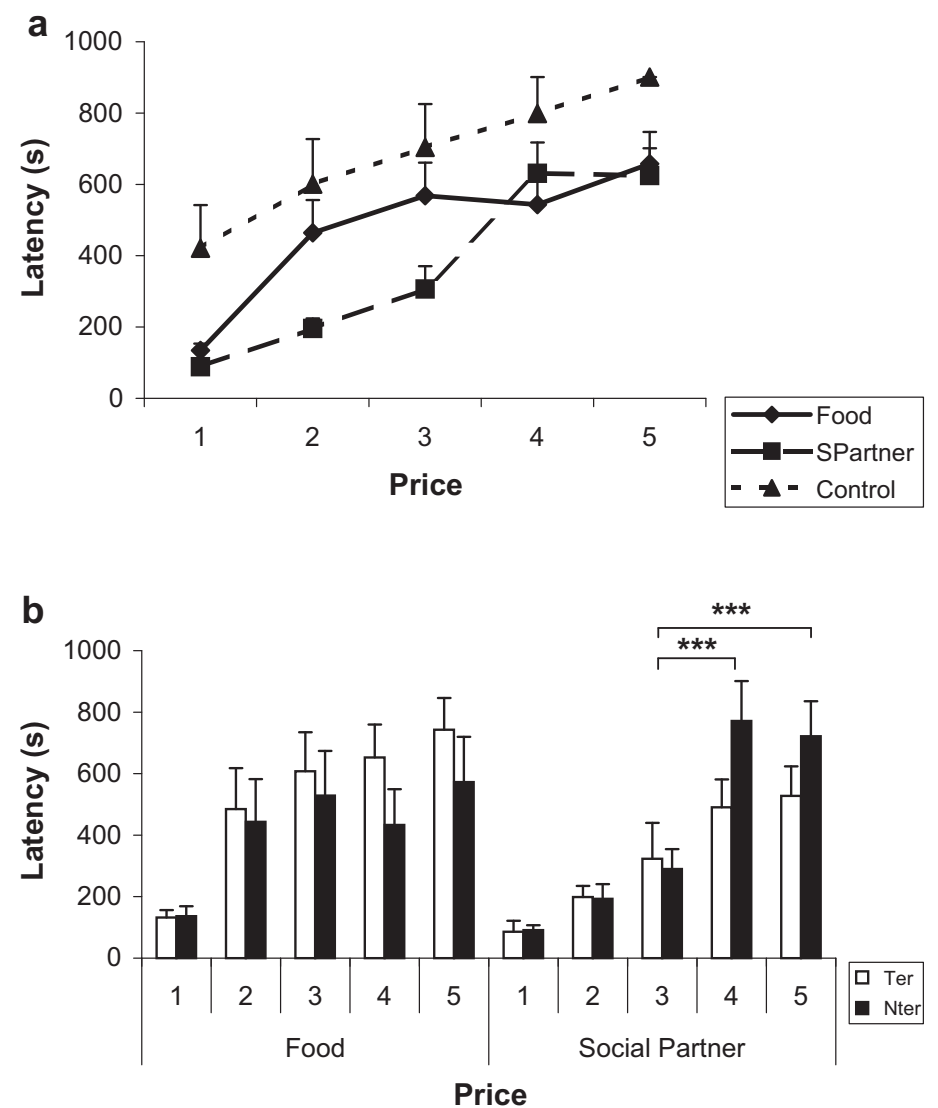

Fig. 2. Latency (s) for opening the door as price increases (a) with different reinforcers: food $(n=12)$, social partner $(n=12)$ and control $(n=5)$ and (b) in territorial and non-territorial males with food and social partner $(n=12) .{ }^{* * *} P<0.001$.
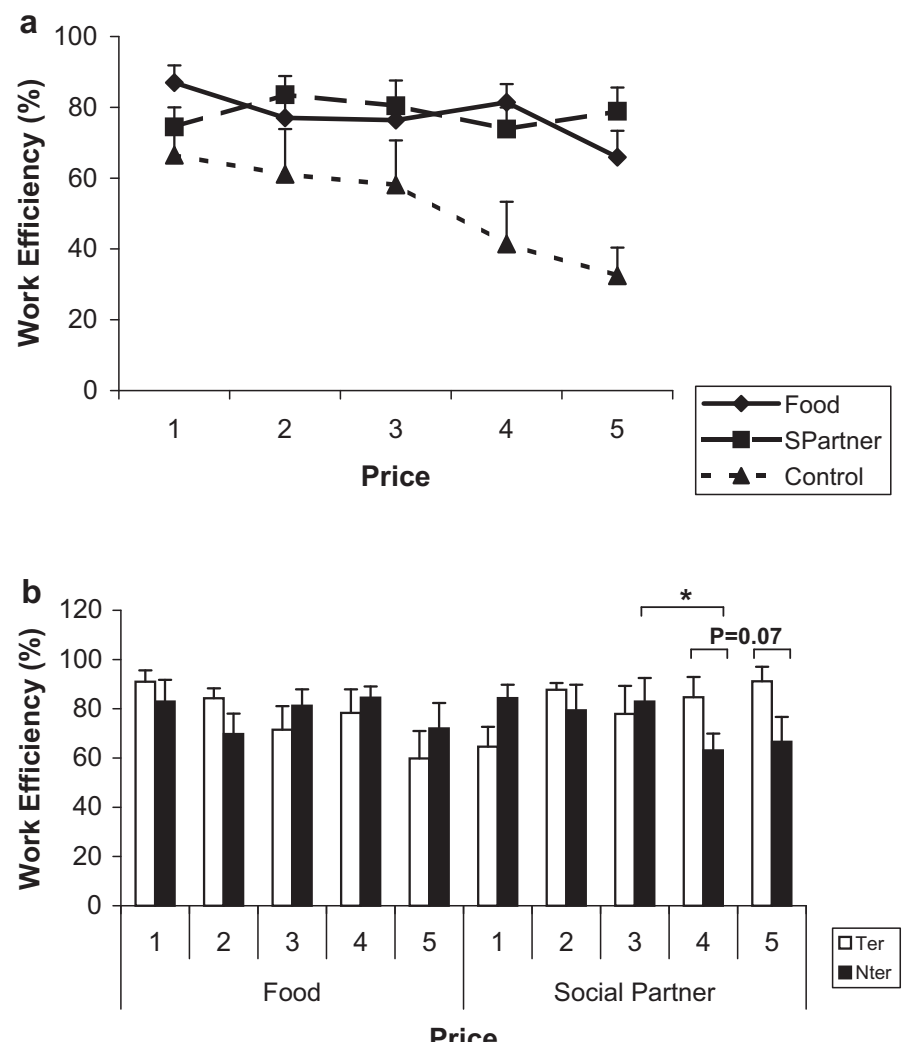

Fig. 3. Work efficiency (\%) for opening the door as price increases (a) with different reinforcers: food $(n=12)$, social partner $(n=12)$ and control $(n=5)$ and (b) in territorial and non-territorial males with food and social partner $(n=12) .{ }^{*} P<0.05$. 
a

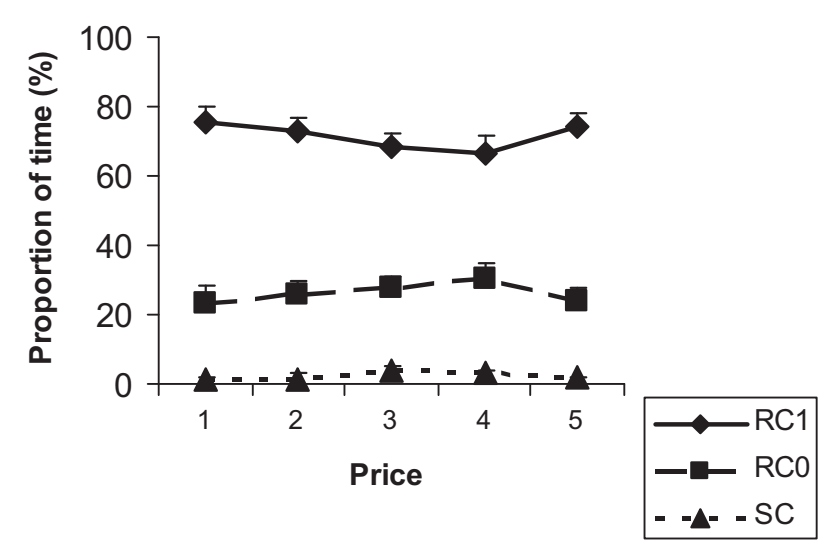

b

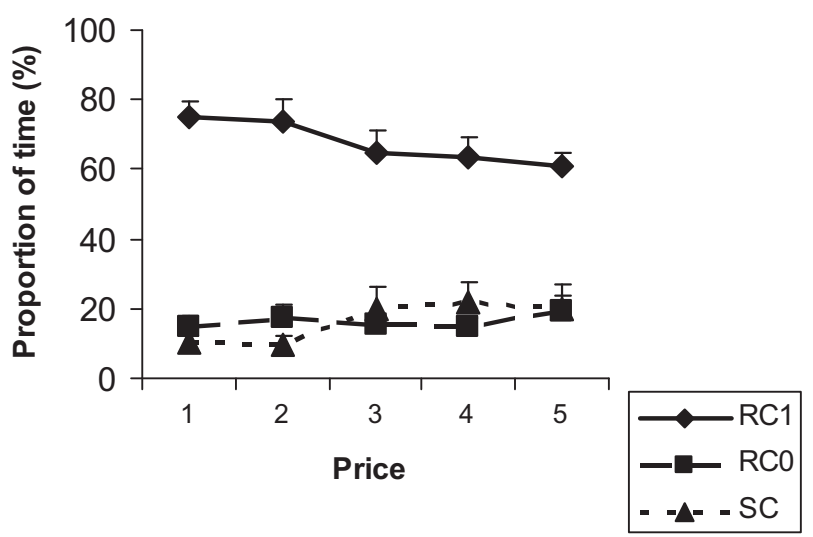

Fig. 4. Proportion of time (\%) spent in area 1 of resource compartment $\left(\mathrm{RC}_{1}\right)$, area 0 of resource compartment ( $\left.\mathrm{RC} \mathrm{C}_{0}\right)$ and in the start compartment ( $\mathrm{SC}$ ) during the period of access to the reinforcer (a) social partner $(n=12)$ (b) control $(n=5)$.

\section{Discussion}

Latency to open the door increased with access cost for all resources, with the highest latency linked to the control reinforcer. Work attention was higher and constant for the social partner and food, and lower and decreasing with increasing price for the control reward. Maximum price paid was consistent with these results, being higher for the social partner and food than for the control reinforcer. Nonterritorial males seemed less willing to work at higher costs for access to the social partner.

\subsection{The push-door, behavioural strategies to open it and reinforcement schedule}

As far as we are aware, this study is the first that used a push-door to quantify motivation in fish. The results suggest that the adapted push-door is an operant paradigm potentially successful for Mozambique tilapia. Animals easily became familiar with the door and quickly adjusted to the task. They developed two spontaneous behavioural responses that may correspond to a behavioural continuum (touches and pushes at the door). One of the criticisms of motivational tests is related to the very extensive and complex training protocols, which may jeopardise the original objective of these tests, the measurement of spontaneous motivation (Würbel, 2009). For this reason, we decided to keep training to the minimum necessary to ensure that animals did understand that their own behaviour towards the door could open it. Therefore touches and pushes were both accepted as work developed at the door. Despite the decision to manually open the door at certain fixed costs, some males did push it strongly enough to open and pass (amplitude of push $\geq 45^{\circ}$, Table 1 ).

When the reinforcer was food, more animals opened the door through pushes than touches. The social partner caused a visible decrease in number of animals opening the door through pushes, and the control resource was never accessed by pushes. It is possible that the access for food triggered a more intense physical reaction in the fish. This does not mean that they worked faster or paid more attention to the task of accessing food, as latency and work attention showed, and they were not necessarily more motivated to access food. This preference for pushing for food may be because that they were trained exclusively with food. Thus, they may have had an additional opportunity to learn a more intense physical response related to the acquisition of this particular reinforcer.

The reinforcement schedules were selected on the basis of a previous pilot study and were confirmed to be well adjusted to the aim. The adopted measures of motivation showed that animals progressively and consistently adjusted their behaviour to increased costs. It is possible that some males could have opened doors at even higher costs than those imposed. However, the inclusion of more trials in the experiment would be too time-consuming for the objectives of the present study.

\subsection{Open vs. closed economy, training and rewards}

The three reinforcers used (female, food and additional space with substrate only) are all potential resources for the ecology and welfare of this species (Galhardo et al., 2008; Galhardo et al., 2009). The present study was developed in an open economy set up, despite the acknowledged criticisms in the validity of open economies to measure motivation (Mason et al., 1998b; Kirkden and Pajor, 2006). The aim was to test the usefulness of the push-door for this species and not to establish definitive comparisons between resources. Therefore, the main priority was to design a relatively simple apparatus and keep the animals non-stressed, thus allowing them to handle the task as well as possible. A closed economy would have required maintaining the animals in social isolation during the study or to design an apparatus in which the animals could live, as in Mason's studies (e.g. Mason et al., 2001; Hovland et al., 2006). This was not feasible without first validating the use of the adapted push-doors. A previous pilot study showed fish did not pay attention to the push-door after a period of social deprivation (some hours to 2 days). Some authors have already shown that fish learning can be affected by stress and high levels of cortisol (Moreira et al., 2004; Moreira and Volpato, 2004; Barreto et al., 2006). This is in line with recent studies where males of this species have 
shown the highest cortisol levels in result of social deprivation (Galhardo et al., submitted). The potential detrimental effects of social isolation for operant tasks were also discussed for pigs (Pedersen et al., 2002). An open economy was also adopted for the control reinforcer (substrate only) to avoid changing the stability of the home tanks by removing the substrate (Galhardo et al., 2008). The food reinforcer was tested in a less open economy as the daily amount of food was delivered exclusively during the trials. However, the animals could still nip particles of food in the substrate when back in their home tanks. The constraints of the present experimental design were taken into consideration when interpreting the behavioural results regarding access to the three reinforcers. It is clear that fish were able to rank reinforcers, as social partner and food seemed more attractive than substrate only, but conclusions about the absolute value of these resources can only be drawn with further refinement.

The adopted training method was very successful in controlling fear and in getting animals familiar with the operant task. However, the decision of using food as the training reinforcer had two main disadvantages. Firstly, fish that were less motivated to eat could have presented unnecessary difficulties during training. Secondly, using food as a training reinforcer and testing it later with other rewards, this resource may have been favoured over others. Training sessions involving the simultaneous use of all rewards under test (if compatible) could be a strategy to take maximum advantage of individual motivations without favouring any reward in particular. At the same time, such a strategy could function as a particularly attractive stimulus which is welcome in training sessions.

A caution note should be added in relation to using food as a yardstick, beyond the aspects already discussed by Hovland et al. (2007) on how food is presented. In studies involving mammals and birds, this resource is usually assumed as the most inelastic, against which motivation for access to other resources is compared (Dawkins, 1990; Kirkden and Pajor, 2006). However, this is not necessarily the case for fish due to a very different metabolism, where food is frequently not the most limiting need (Monaghan, 1990). A high priority to eat is both a species-dependent issue, and can vary with different life stages. For example, during breeding in natural conditions, Mozambique tilapia do not eat or can go for long periods without food (Neil, 1966). Therefore, it is assumed that motivation to breed may decrease motivation to feed in this species.

Reward size in this experiment was related to the maintenance of its attractiveness throughout the trials. This was because the objective was to measure access to reward and not reward consumption (demand measures). In relation to food, the aim was to keep the usual meal size per trial session. This approach did not satiate the animals, but given the usual routine, it was also unlike to frustrate them. Instead of investing in quantity, an effort was made to manipulate the fish positive perception about food (see Section 2 for details, Galhardo and Oliveira, 2009). In relation to access to the social partner, 30 min of free contact with the female was judged to be enough time to avoid aversive interruption of social interactions. A possible consequence of this unconstrained interaction was that consumption in relation to the female did not change with access cost. However, it is interesting to note that once with the female, males no longer left the resource compartment (swimming back to the start compartment). In contrast, when tested for the control (additional space/substrate), males returned to the start compartment a number of times, possibly because they valued this extra space (SC). In any case, all males preferred to stay in the area with substrate. For a more complete understanding of behaviour with the reward, it would have been useful to make behavioural samples for the complete period of $30 \mathrm{~min}$, and not only for the first $10 \mathrm{~min}$, as was the case.

\subsection{Measures of motivation}

The three measures of motivation (latency, work attention and maximum price paid) were chosen as the most appropriate indices to measure access to a reward with regard to our experimental design. All of them distinguished between social partner/food rewards and substrate (control) in a consistent way. The increased latencies for the control (additional space with substrate only) suggested that animals worked harder for the social partner and food access, thus preferring these resources. In order to avoid inappropriate analysis of motivation based upon work effort when two different behaviours were involved (pushes and touches), 'work attention' was adopted as a measure of motivation. This parameter measured the degree of attention and investment at the door regardless the type of behaviour the animals engaged in. Results have shown that work attention was similar regarding social partners and food and lower to a substrate-only compartment, i.e. the social partner and food attracted more attention and made them develop a more prolonged behavioural response than substrate-only. Data on maximum price paid also supports this. It should be noted that once animals stopped opening the door, they would not open it again in subsequent trials (except for two animals, out of 12), which made this index quite consistent. In any case, measure of maximum price paid was limited by a ceiling effect in the sense that some males still worked the door at $200 \mathrm{~g}$ (maximum price imposed by the experimental design). However, for the control (substrate only), efforts to open the door did not exceed $100 \mathrm{~g}$.

Social status influenced the motivation indices to have access to the social partner: the non-territorial males showed higher latencies and lower work attention at higher costs. On the other hand, territorial males interacted more with the females at any costs. Therefore, the results consistently suggest that territorial males were more motivated to have access to females at higher costs than non-territorial males.

\section{Conclusion}

The existence of a paradigm to quantify the value fish attach to certain resources is potentially very useful in a number of behavioural, physiological, cognitive and welfare studies. Information derived from these studies also has relevant implications for the management of their wel- 
fare in captivity, namely in the identification of relevant needs.

The present study has shown that the push-door can be used to measure motivation for access to resources in this species. In future studies the experimental set up can be refined in order to increase validity of motivation measures in relation to the present and other resources. The adaptation to an automated, computer controlled pushdoor is one of the aspects to improve, as it can reduce sampling effort. In this way, sampling sizes can increase, which is important for better interpretation of individual differences. To test the animals in a closed economy and deal with all the resources in the same way and, refine the reward sizes are important steps towards a more precise measurement of motivation. It is also relevant to keep the animals healthy, unstressed and appropriately trained. Provided these aspects are taken into consideration, the push-door can successfully measure motivation in this species.

\section{Acknowledgments}

The authors wish to thank Anna Olsson for previous discussions on how to measure motivation, Chris Noble for the English revision of the manuscript and David Gonçalves for the statistical analysis. We are also very grateful to Anna Olsson, Stephanie Yue and Humberto Rosa for their comments on this manuscript. This study was funded by the Pluriannual Program of Fundação para a Ciência e a Tecnologia (FCT, UI\&D 331/2001) and L.G. was supported by a PhD fellowship from FCT (SFRH/BD/16162/2004).

\section{Appendix A. Supplementary data}

Supplementary data associated with this article can be found, in the online version, at doi:10.1016/j.applanim.2010.12.008.

\section{References}

Antunes, R.A., Oliveira, R.F., 2009. Hormonal anticipation of territorial challenges in cichlid fish. Proc. Natl. Acad. Sci. U.S.A. Biol. Sci. 106, 15985-15989.

Barreto, R.E., Volpato, G.L., Pottinger, T.G., 2006. The effect of elevated blood cortisol levels on the extinction of a conditioned stress response in rainbow trout. Horm. Behav. 50, 484-488.

Cooper, J.J., Appleby, M.C., 2003. The value of environmental resources to domestic hens: a comparison of the work-rate for food and for nests as a function of time. Anim. Welfare 12, 39-52.

Dawkins, M.S., 1983. Battery hens name their price - consumer demand theory and the measurement of ethological needs. Anim. Behav. 31, 1195-1205.

Dawkins, M.S., 1990. From an animals point of view - motivation, fitness, and animal-welfare. Behav. Brain Sci. 13, 1-61.

Duncan, I.J.H., 2006. The changing concept of animal sentience. Appl. Anim. Behav. Sci. 100, 11-19.

Duncan, I.J.H., Kite, V.G., 1987. Nest site selection and nest-building behaviour in domestic fowl. Anim. Behav. 37, 215-231.

Dunlop, R., Millsopp, S., Laming, P., 2006. Avoidance learning in goldfish (Carassius auratus) and trout (Oncorhynchus mykiss) and implications for Pain perception. Appl. Anim. Behav. Sci. 97, 255-271.

Endo, M., Kumahara, C., Yoshida, T., Tabata, M., 2002. Reduced stress and increased immune responses in Nile tilapia kept under self-feeding conditions. Fisheries Sci. 68, 253-257.

Galhardo, L., Almeida, O., Oliveira, R.F., 2009. Preference for the presence of substrate in male cichlid fish: effects of social dominance and context. Appl. Anim. Behav. Sci. 120, 224-230.
Galhardo, L., Correia, J., Oliveira, R.F., 2008. The effect of substrate availability on behavioural and physiological indicators of welfare in the African cichlid (Oreochromis mossambicus). Anim. Welfare 17, 239-254.

Galhardo, L., Oliveira, R.F., 2009. Psychological stress and welfare in fish. ARBS Annu. Rev. Biomed. Sci. 11, 1-20.

Hansen, S.W., Jensen, M.B., 2006. Quantitative evaluation of the motivation to access a running wheel or a water bath in farm mink. Appl. Anim. Behav. Sci. 98, 127-144.

Herrero, M.J., Pascual, M., Madrid, J.A., Sanchez-Vazquez, F.J., 2005. Demand-feeding rhythms and feeding-entrainment of locomotor activity rhythms in tench (Tinca tinca). Physiol. Behav. 84, 595-605.

Hogan, J.A., Kleist, S., Hutching, Cs, 1970. Display and food as reinforcers in Siamese Fighting Fish (Betta splendens). J. Comp. Physiol. Psychol. $70,351-\&$

Hovland, A.L., Mason, G., Ahlstrom, O., Bakken, M., 2007. Responses of farmed silver foxes (Vulpes vulpes) to excessive food availability: implications for using food as a yardstick resource in motivation tests. Appl. Anim. Behav. Sci. 108, $170-178$.

Hovland, A.L., Mason, G., Boe, K.E., Steinheim, G., Bakken, M., 2006. Evaluation of the 'maximum price paid' as an index of motivational strength for farmed silver foxes (Vulpes vulpes). Appl. Anim. Behav. Sci. 100, 258-279.

Huntingford, F.A., Adams, C., Braithwaite, V.A., Kadri, S., Pottinger, T.G., Sandoe, P., Turnbull, J.F., 2006. Current issues in fish welfare. J. Fish Biol. 68, 332-372.

Kirkden, R.D., Edwards, J.S.S., Broom, D.M., 2003. A theoretical comparison of the consumer surplus and the elasticities of demand as measures of motivational strength. Anim. Behav. 65, 157-178.

Kirkden, R.D., Pajor, E.A., 2006. Using preference, motivation and aversion tests to ask scientific questions about animals' feelings. Appl. Anim. Behav. Sci. 100, 29-47.

Lea, S.E.G., 1978. The psychology and economics of demand. Psychol. Bull. $85,441-466$.

Martin, P., Bateson, P., 2007. Measuring Behaviour: an Introductory Guide. Cambridge University Press, Cambridge.

Mason, G., Garner, J., McFarland, D., 1998a. Assessing animal priorities: future directions. Anim. Behav. 55, 1082-1083.

Mason, G., McFarland, D., Garner, J., 1998b. A demanding task: using economic techniques to assess animal priorities. Anim. Behav. 55, 1071-1075.

Mason, G.J., Cooper, J., Clarebrough, C., 2001. Frustrations of fur-farmed mink. Nature 410, 35-36.

Matthews, L.R., Ladewig, J., 1994. Environmental requirements of pigs measured by behavioral demand-functions. Anim. Behav. 47, 713-719.

Millsopp, S., Laming, P., 2008. Trade-offs between feeding and shock avoidance in goldfish (Carassius auratus). Appl. Anim. Behav. Sci. 113, 247-254.

Monaghan, P., 1990. Consumer demand: can we deal with different priorities? Behav. Brain Sci. 13, 29-30.

Moreira, P.S.A., Pulman, K.G.T., Pottinger, T.G., 2004. Extinction of a conditioned response in rainbow trout selected for high or low responsiveness to stress. Horm. Behav. 46, 450-457.

Moreira, P.S.A., Volpato, G.L., 2004. Conditioning of stress in Nile tilapia. J. Fish Biol. 64, 961-969.

Neil, E.H., 1966. Observations on behavior of Tilapia Mossambica (Pisces Cichlidae) in Hawaiian ponds. Copeia 1, 50-56.

Oliveira, R.F., Almada, V.C., 1996. Dominance hierarchies and social structure in captive groups of the Mozambique tilapia Oreochromis mossambicus (Teleostei: Cichlidae). Ethol. Ecol. Evol. 8, 39-55.

Oliveira, R.F., Almada, V.C., 1998. Dynamics of social interactions during group formation in males of the cichlid fish Oreochromis mossambicus. Acta Ethol. 1, 57-70.

Olsson, I.A.S., Keeling, L.J., 2002. The push-door for measuring motivation in hens: laying hens are motivated to perch at night. Anim. Welfare $11,11-19$.

Olsson, I.A.S., Keeling, L.J., McAdie, T.M., 2002. The push-door for measuring motivation in hens: an adaptation and a critical discussion of the method. Anim. Welfare 11, 1-10.

Pedersen, L.J., Jensen, M.B., Hansen, S.W., Munksgaard, L., Ladewig, J., Matthews, L., 2002. Social isolation affects the motivation to work for food and straw in pigs as measured by operant conditioning techniques. Appl. Anim. Behav. Sci. 77, 295-309.

Petherick, J.C., Rutter, S.M., 1990. Quantifying motivation using a computer-controlled push-door. Appl. Anim. Behav. Sci. 27, 159-167. 
Prescott, M.J., Buchanan-Smith, H.M., 2003. Training non-human primates using positive reinforcement techniques. Appl. Anim. Behav. Sci. 6, 157-161.

Sherwin, C.M., Nicol, C.J., 1996. Reorganization of behaviour in laboratory mice, Mus musculus, with varying cost of access to resources. Anim. Behav. 51, 1087-1093.

Volpato, G.L., Goncalves-de-Freitas, E., Fernandes-de-Castilho, M., 2007. Insights into the concept of fish welfare. Dis. Aquat. Organ. 75, 165-171.
Widowski, T.M., Duncan, I.J.H., 2000. Working for a dustbath: are hens increasing pleasure rather than reducing suffering? Appl. Anim. Behav. Sci. 68, 39-53.

Würbel, H., 2009. Ethology applied to animal ethics. Appl. Anim. Behav. Sci. 118, 118-127.

Yue, S., Duncan, I.J.H., Moccia, R.D., 2008. Investigating fear in rainbow trout (Oncorhynchus mykiss) using the conditioned-suppression paradigm. J. Appl. Anim. Welfare Sci. 11, 14-27.

Zar, J.H., 1984. Biostatistical Analysis. Prentice-Hall, Englewood Cliffs, N.J. 